Ferro, D.A., Deijen, J.B., Koppes, L.L., Mechelen, W. van, Twisk, J.W., Drent, M.L. The effects off physical activity and fitness in Adolescence on cognition in adulthood and the role of insulinlike growth factor I . Journal of Physical Activity \& Health: 2016, 13(4), 392-402

\begin{tabular}{|l|l|}
\hline $\begin{array}{l}\text { Postprint } \\
\text { Version }\end{array}$ & 1.0 \\
\hline Journal website & http://journals.humankinetics.com/doi/10.1123/ipah.2014-0594 \\
\hline Pubmed link & $\underline{\text { http://www.ncbi.nlm.nih.gov/pubmed/26389609 }}$ \\
\hline DOI & $10.1123 /$ jpah.2014-0594 \\
\hline
\end{tabular}

This is a NIVEL certified Post Print, more info at http://www.nivel.eu

\title{
The Effects of Physical Activity and Fitness in Adolescence on Cognition in Adulthood and the Role of Insulin-Like Growth Factor I
}

\author{
Doeschka A. Ferro, Jan Berend DeiJen, Lando L. Koppes, Willem VAn Mechelen, \\ JOS W. TWISK, AND MADELEINE L. DRENT
}

\begin{abstract}
Background: Physical activity and fitness in adolescence may improve cognition in adulthood by increasing insulin-like growth factor I (IGF-I). Methods: As part of the Amsterdam Growth and Health Longitudinal Study, following subjects from age 13 to 42 years, physical activity and fitness of 303 subjects were assessed annually between the ages 13 to 16 . At mean age 36, physical activity, fitness and IGF-I were measured. At mean age 42, IGF-I and cognitive factors (ie, executive functioning and visual-spatial memory) were measured. The linear regression of physical activity and fitness in adolescence and IGF-I in adulthood on cognitive scores in adulthood was investigated. Results: A significant association was found in males between physical activity in adolescence and executive function in adulthood (Spatial Working Memory Between Errors: $\beta$ $=-.18, \mathrm{~B}=-.13,95 \% \mathrm{CI}=-.259$ to -.010 ; Spatial Working Memory Strategy: $\beta=-.20, \mathrm{~B}=-.08,95 \% \mathrm{CI}=-.147$ to -.014$)$. No association between physical activity or fitness in adolescence and cognitive function in adulthood was found in females, nor any intermediate role for IGF-I in either sex. Conclusions: The results suggest a stimulating effect of adolescent physical activity in males on executive functions in adulthood, emphasizing the importance of an active lifestyle among adolescent males.
\end{abstract}

In the past decades empirical evidence has become available to confirm the general benefits of physical activity and fitness on health. A part of research in this field has been directed to the role of physical activity as well as fitness in cognition. Physical activity can be defined as the amount of motor actions performed, meaning bodily movement produced by skeletal muscles that results in energy expenditure. 1 In contrast to physical activity, which represents bodily movement, physical fitness represents a physiological status defined by the amount of oxygen the body can transport and use which can be measured by lung function capacity.. Cognition can be defined as processes of knowing, including attending, remembering, and reasoning.2 A higher level of engagement in physical activities seems protective 
Ferro, D.A., Deijen, J.B., Koppes, L.L., Mechelen, W. van, Twisk, J.W., Drent, M.L. The effects of physical activity and fitness in Adolescence on cognition in adulthood and the role of insulinlike growth factor I . Journal of Physical Activity \& Health: 2016, 13(4), 392-402

against cognitive decline and may reduce the risk of dementia.3 Meanwhile, an inactive lifestyle is associated with cognitive decline.4 Different levels of decline of cognition may progressively affect performance of activities of daily living (ADL). Mild cognitive impairment negatively influences an individual's ability to perform complex ADL, such as household activities, handling money, shopping, and transportation. Dementia initially affects people's ability to perform complex activities of daily life, but later in the disease course disabilities progress to more basic activities such as dressing and toileting.s Physical activity can lay out a neural architecture providing a cognitive reserve, which can be defined as the capacity of the brain to cope with brain pathology to minimize or postpone the onset of clinical symptoms.6 In addition, aspects of life experiences, like engagement in leisure activities, may result in functionally more efficient cognitive networks and therefore provide a cognitive reserve that delays the onset of clinical manifestations of agerelated cognitive deficits and even dementia, 7 thereby slowing down pathology at older age. 8 Results from epidemiologic studies suggest that experiences at all stages, even in late life, can increase this reserve. 9 The majority of studies on cognition and physical activity have focused on the reduction of cognitive deterioration in elderly.10 Recently, 4 longitudinal studies were analyzed with follow ups after 8 to 18 years.11 Physically active elderly were found to perform better at reasoning and fluency tasks than their sedentary peers and additionally suffered less decline in fluency after follow up.

Word fluency requires the generation of multiple single words from a single cue within a given time, which is thought to be a frontal lobe process.12 With respect to younger subjects, studies examined the relationship between physical activity and cognitive performance, including school achievements in elementary school children. For instance, physically active lessons of moderate intensity improved overall performance on a standardized test of school achievement by $6 \%$ compared with a decrease of $1 \%$ for controls, as was indicated by the results of a 3-year cluster randomized, controlled trial in 24 elementary schools.13 In another study, an association was observed between physical activity at age 11 and attention at ages 11 and 13 years with data from 4755 children. Results indicated that moderate-tovigorous physical activity might be beneficial for attention processes in adolescence. 14 To examine the effect of aerobic fitness on cognitive performance, a meta-analysis was performed on 37 studies including subjects with ages from 11 to 83 years. Samples were categorized as children and young adults (5-29 yrs), adults (30-59 yrs), and older adults (over $60 \mathrm{yrs}$ ). 7 The results of the cross-sectional studies indicated that the fit individuals showed a better cognition than the unfit individuals. In addition, for the cross-sectional studies, aerobic fitness was negatively predictive of cognitive performance for children and young adults, positively predictive for adults, and not significantly related for older adults. For the pre-post comparisons, aerobic fitness was negatively predictive of cognitive performance for older adults, but was not a significant predictor for the other 2 age groups. As these inconsistent results do not support a mediating role of aerobic fitness, the authors suggest that variables other than aerobic fitness (both physiological and psychological) may explain the positive relationship between physical activity and cognition.

In a study on the long-term effects of activity between the ages of 15 and 25 on cognitive performance in older age ( 55 to 85 years), men who had exercised with low intensity were found to exhibit a higher information processing speed at old age than 
Ferro, D.A., Deijen, J.B., Koppes, L.L., Mechelen, W. van, Twisk, J.W., Drent, M.L. The effects of physical activity and fitness in Adolescence on cognition in adulthood and the role of insulinlike growth factor I . Journal of Physical Activity \& Health: 2016, 13(4), 392-402

men who had either vigorously exercised or not at all.15 In another study, females who had been active throughout their teenage years were found to show a lower incidence of cognitive impairment around the age of 70.16 Studies in young adulthood showed that physical activity at age 36 was associated with a slower rate of decline in memory after 7 and 17 years. 17 In contrast, after a 10 year timeframe the selfreported physical activity of middle-aged civil servants was associated with fluid intelligence and phonemic fluency, but not short-term memory.18 Executive functioning, reflecting a mental process guiding cognitive control for purposeful behavior, 19 speed of information processing as well as memory in later life (mean age 76 years) have been found to be positively associated with physical activity previously in midlife (at a mean of age 51 years).20 The available data on the relationship between physical activity and cognition are conflicting with respect to the level of activity needed to improve cognitive function. The results of a metaanalysis in nondemented subjects followed for 1 to 12 years indicated that, relative to subjects being sedentary, subjects reporting a high level of activity had a $38 \%$ reduced risk of cognitive decline, while lowto- moderate levels of activity reduced the risk of cognitive decline with $35 \%$. Thus, estimates of association were similar for high and low-to-moderate levels of activity. In contrast to the cited study above, 15 all levels of physical activity seem to reduce the risk of cognitive decline. $21 \mathrm{~A}$ possible mediating factor explaining the effects of physical activity on the brain are hormones. Several studies have hinted at a mediating role for insulin-like growth factor I (IGF-I) between physical fitness and cognitive performance in humans.22 There is a body of evidence that higher IGF-I levels in healthy individuals and in distinct patient groups are associated with a better cognitive performance. ${ }_{23} \mathrm{In}$ addition, IGF-I production is stimulated by physical exercise 24 and fitter subjects are found to have higher levels of circulating IGF-I.25 The circulating IGF-I can cross the blood-brain barrier and may eventually improve cognitive functions.

${ }_{23}$ The increase of IGF-1 levels by exercise may be enduring as a consequence of functionally relevant (epigenetic) changes to the IGF-I gene.26-28 The aim of this study was to examine the effects of adolescent physical activity and fitness on cognitive function in adulthood. It is hypothesized that more active and/or more fit adolescents will have better memory and executive function in adulthood. As the production of IGF-I is stimulated by physical activity and enhanced in persons with higher fitness levels, it is theorized that IGF-I is one of the mediating factors between adolescent activity and adult cognitive function.

\section{METHODS}

\section{Participants}

A number of 303 participants of the Amsterdam Growth and Health Longitudinal Study (AGAHLS) were included in the current study.

The sample consisted of healthy, Caucasian subjects of whom 143 were male and 160 female. In 1976 this study was started to gain an understanding of growth, development and health of children going into puberty and included 698 secondary school pupils aged 13.29 Data concerning health, lifestyle and psychological factors were collected. Four annual measurements of physical activity and fitness were carried out between the ages 13 and 16 (SD 0.6). In adulthood, physical activity, fitness and IGF-I were examined at mean age of 36 (range 34 to 38, SD 0.7). In addition, at mean age 42 (range 41 to 46, SD 0.7) IGF-I was determined and 
Ferro, D.A., Deijen, J.B., Koppes, L.L., Mechelen, W. van, Twisk, J.W., Drent, M.L. The effects of physical activity and fitness in Adolescence on cognition in adulthood and the role of insulinlike growth factor I . Journal of Physical Activity \& Health: 2016, 13(4), 392-402

cognitive tests administered. For the current study, subjects were selected on the basis of having at least 1 physical activity and fitness measure taken in adolescence and completion of cognitive tests and at least 1 IGF-I sample taken in adulthood. The socioeconomic levels were obtained using standardized questionnaires and turned out to be slightly above the Dutch national average (data not shown). All participants had given written consent and the study was approved by the Medical Ethical Committee at VU University Medical Center.

\section{Measurements}

\section{Physical Activity.}

Physical activity was a self-reported measure in metabolic equivalents (MET) using a standardized activity interview based on a questionnaire. ${ }_{30,31}$ One MET is defined as the oxygen uptake of a person sitting at rest. This is equivalent to a body oxygen consumption of approximately $3.5 \mathrm{ml}$ per kilogram of body weight per minute for an average adult. Subjects reported their physical activities of the previous 3 months. They documented their work and home activities, their involvement in organized as well as in unorganized sports activities and their physical activity while traveling. For example, subjects were asked "Are you member of a sport club?"; "What is the average active time per training?"; "What is the number of training sessions per week?" The investigators took only those activities into account, which had a duration of at least $5 \mathrm{~min}$ and an intensity level of more than 4 times the basal metabolic rate. As children aged 13 years may alternate activities of a short duration, the 5 min criterion was chosen to reduce the risk of underestimation of the total amount of activity.

The physical activities were classified as light (4-7 METs), medium-heavy (7-10 METs), and heavy (>10 METs). For the purpose of scoring, the METs assigned to these categories were 5.5, 8.5 and 11.5, respectively. The interview assessed the average weekly time spent doing activities in each of the 3 categories.

MET scores per week were derived through a multiplication of the average time spent per week ( $\mathrm{min}$ ) in each category with the respective MET value for that category. The scores of the 3 levels were added to arrive to a score of total METs times minutes per week: Weighted Activity Score $=(5.5 \times \mathrm{min} /$ week $)+(8.5 x$ $\mathrm{min} /$ week $)+(11.5 \times \mathrm{min} /$ week $)$, in METs/week. Thus, the weighted activity score is a combined measure of the duration and intensity of the daily physical activity.32

\section{Physical Fitness.}

Physical fitness was defined as the maximal oxygen uptake $\left(\mathrm{VO}_{2} \max \right)$ on a treadmill running test (Quinton, model 18-54). Participants were equipped with a mouthpiece to collect the expired gas and a nose clip. After a short practice phase serving as warming-up and to familiarize with the apparatus, subjects were verbally encouraged to run at a constant speed of $8 \mathrm{~km} / \mathrm{h}$.

Meanwhile, the slope of the treadmill was increased $2.5 \%$ every 2 minutes until complete exhaustion had been reached. Measurements of $\mathrm{VO}_{2}$ max were taken using the Ergoanalyzer (Jaeger, Bunnik, The Netherlands) and were corrected for body size. The effectiveness of this treadmill test in reaching a true maximal oxygen uptake was evaluated from the maximal heart rate (more than $95 \%$ of the predicted maximum corrected for age) and the respiratory gas exchange ratio (a value of more 
Ferro, D.A., Deijen, J.B., Koppes, L.L., Mechelen, W. van, Twisk, J.W., Drent, M.L. The effects of physical activity and fitness in Adolescence on cognition in adulthood and the role of insulinlike growth factor I . Journal of Physical Activity \& Health: 2016, 13(4), 392-402

than 1.0). More details on the physical activity and the physical fitness measures can be found in Kemper et al.29

\section{Cognitive Tests.}

Two subtests, the Paired Associates Learning (PAL) task and the Spatial Working Memory (SWM) task, from the Cambridge Neuropsychological Test Automated Battery (CANTAB) ${ }_{33}$ were administered. Subjects were tested individually in a sound attenuated room at the VU University during approximately $30 \mathrm{~min}$. The PAL subtest is a visual-spatial memory task relying on medial temporal lobe functioning. Six to eight boxes are presented at the same time on the screen and are opened in a randomized order. One or more of them will contain an image (colored geometrical shape). At the end of the trial, subjects are shown an image, one at a time, in the middle of the screen and asked to touch the box where the pattern was originally located. If the participant makes an error, the patterns are represented (up to 6 times) to remind the participant of their locations. The number of images increases up to 8 in progressive stages. A subject progresses to the next stage if all the locations are correctly remembered. The test is terminated when a stage cannot be completed. From the total of 21 outcome measures 2 outcome measures were selected on the basis of having sufficient spread in scores between subjects. The First Trial Memory Score (FTMS) was the number of patterns correctly located on the first trial across all stages, with higher scores reflecting better performance. The Total Number of Errors (TE) was the adjusted number of errors for the completed stages, so that more errors signified a poorer performance.

The SWM task was used to determine working memory and strategic memory control, that is executive function which relies strongly on frontal lobe functioning. Several boxes were presented on screen and participants could open the boxes to find a token that was hidden. After the token had been found, they had to search for a new token in the other unopened boxes. A self-ordered search strategy has to be applied, which relies on intact frontal lobe and executive functioning. From the total of 17 outcome measures the between errors (BE) and strategy scores were recorded. The amount of between errors was the number of times a box was opened in which a token had already been found. Accordingly, more errors demonstrated worse spatial memory. Strategy scores were calculated on the basis of the number of times a subject began the search with a different box and low strategy scores reflect the use of the efficient strategy of starting at the same box (see CANTABeclipse Test Administration Guide). .34 The CANTAB tests were conducted using an Intel Pentium III processor and a 17' ELO touch screen. The distance between the touch screen and participants was set at $40 \mathrm{~cm}$.

\section{General Cognitive Ability.}

The Dutch Adult Reading Test (DART), administered at age 42, is the Dutch version of the National Adult Reading Test (NART). ${ }_{35}$ Participants were required to correctly pronounce a list of 50 irregular Dutch words. The score is an estimate of verbal intelligence, resilient to cognitive decline. $36 \mathrm{~A}$ higher score means a better performance. In the current study, the score on the DART serves as an estimate for general cognitive ability to correct for preexisting differences in cognitive capacity between subjects. 
Ferro, D.A., Deijen, J.B., Koppes, L.L., Mechelen, W. van, Twisk, J.W., Drent, M.L. The effects of physical activity and fitness in Adolescence on cognition in adulthood and the role of insulin like growth factor I. Journal of Physical Activity \& Health: 2016, 13(4), 392-402

\section{$I G F-I$.}

Venous blood was drawn from the antecubital vein to determine IGF-I serum levels using a commercially available assay (Chemiluminescent immunometric, Immulite 2500, DPC, Los Angeles, USA). The detection limit was $3.2 \mathrm{nmol} / \mathrm{l}$. The intra- and interassay coefficients of variation were $5 \%$ and $8 \%$, respectively, for the entire range $(7-60 \mathrm{nmol} / \mathrm{l})$.

\section{Education.}

Education was graded according to the highest level of completed education. Of the subjects $17 \%$ had finished no further than high school education, $16 \%$ secondary vocational education, $37 \%$ higher vocational education, $21 \%$ academic education and $9 \%$ specified "other."

\section{Data Analysis}

Physical activity and fitness measurements within adolescence (13-16 years of age) were averaged over the 4 annual measurements.

It appeared useful to obtain a single, more reliable estimate for each variable because the stability of physical activity over this period, indicated by the interperiod correlation coefficient (IPC), was found to be moderate in the original sample of the AGAHLS (boys, IPC $=.44$; girls, IPC $=.58$ ). .37 A single outlier was removed from the physical activity data in adolescence, as it was more than 5 SD above the mean and suspect for data entry error. A logarithmic transformation was performed for the PAL TE scores to correct for skewness. There were 26 missing values of physical activity and 19 missing values of fitness in adulthood; however a Missing Values Analyses revealed no differences between the groups with missing values and those with recorded data on the SWM, PAL or METS scores in adolescence. In addition, the extent of missing data were relatively small (ie, less than $10 \%$ ). Thus, replacement of the missing values by the mean can be considered to be appropriate. 38,39 Two scores missing on the DART were also replaced by the mean. As these subjects had average educational levels, it seemed justified to replace the missing values by the mean when entered as covariate. There were 11 missing values of IGF-I at age 36 and 6 missing values at age 42. In case a subject had 1 missing value of IGF-I at age 36, the value of IGF-I at age 42 was taken and vice versa. The mean IGF-I change per year has been estimated to be 3.5 (age $<40$ years) and 2.5 (age 40 to 50 years) $\mathrm{ng} / \mathrm{ml}$ in men, and 2.3 (age $<40$ years) and 2.1 (age 40 to 50 years) $\mathrm{ng} / \mathrm{ml}$ in women.40 Thus, from age 36 to age 42 the estimated IGF-I decline can be calculated as $19(4 \times 3.5+2 \times 2.5) \mathrm{ng} / \mathrm{ml}(2.47 \mathrm{nmol} / \mathrm{l})$ in males and $13.4(4 \times$ $2.3+2 \times 2.1) \mathrm{ng} / \mathrm{ml}(1.74 \mathrm{nmol} / \mathrm{l})$ in females. This is quite in accordance with the IGF-I decline in our sample (see Table 1). As this decline is small as indicated by the effect sizes (males: $d=.41$, females: $d=.29)_{41}$ and IGF-I levels at age 36 and age 42 were highly correlated (males $r=.75$, females $r=.61$ ), IGF-I levels can be considered to be quite stable. IGF-1 at age 36 as well as age 42 were missing for 4 subjects. These 4 missing values were not replaced. Statistical analysis was carried out using SPSS 22. Separate correlations in males and females between IGF-I, physical activity, fitness and cognitive tasks were identified to establish the mediating role of IGF-I.42 Multiple regression analyses were carried out with sex as moderator for physical activity and fitness in adolescence on cognitive scores in adulthood by means of 3 successive models. Model 1 was a crude analysis carried 
Ferro, D.A., Deijen, J.B., Koppes, L.L., Mechelen, W. van, Twisk, J.W., Drent, M.L. The effects off physical activity and fitness in Adolescence on cognition in adulthood and the role of insulinlike growth factor I . Journal of Physical Activity \& Health: 2016, 13(4), 392-402

out separately for physical activity and fitness in adolescence on cognitive scores in adulthood. Model 2 was a model corrected for covariates (education, DART and physical activity or fitness in adulthood). Model 3 was a model included the covariates and IGF-I (average of IGF-I at age 36 and 42). In addition, regression analyses were carried out in the same 3 steps separately for males and females. Statistical results are presented by the standardized regression coefficient $\beta$, the unstandardized regression coefficient B and the $95 \%$ confidence interval (CI). Covariates included were education, DART, and physical activity or fitness in adulthood. Education was used as covariate because it may confound the relationship between physical activity or fitness in adolescence with cognitive performance in adulthood. As scores on the DART serve as an estimate for general cognitive ability, they were included as covariate to correct for preexisting differences in cognitive capacity between subjects. Physical activity in adulthood was used as covariate to correct for its potentially confounding effect on the relationship between physical activity in adolescence and cognitive performance. In addition, fitness in adulthood was used as covariate to correct for its potentially confounding effect on the relationship between fitness in adolescence and cognitive performance in adulthood. Finally, IGF-I was entered as predictor to examine whether this variable plays a role in the relationship between physical activity or fitness and cognitive performance.

\section{RESULTS}

\section{Physical Activity and Fitness}

Table 1 shows the mean physical activity and fitness in adolescence and adulthood. Activity levels of males and females were significantly different in adolescence $\left(t_{(301)}\right.$ $=5.08, P<.0001)$, but were the same in adulthood $\left[t_{(301)}=-1.55, P=.12\right]$. Males had significantly higher fitness levels than females in adolescence $\left[t_{(301)}=17.56 P<\right.$ $.0001]$ as well as in adulthood $\left[t_{(282)}=13.85 P<.0001\right]$.

\section{Cognitive Tests}

Scores of males and females on the cognitive tests are presented in Table 1. On average, females scored significantly better on the DART. Males made significantly less between errors and showed superior strategic skills on the SWM, indicating better working memory performance and executive functioning. No difference between males and females was observed on the performance on the PAL [FTMS $t_{(301)}=.005, P=.97$; TE $(\lg )\left(t_{(301)}=1.12, P=.24\right]$.

\section{IGF-I}

The mean serum IGF-I levels are presented in Table 1. No significant difference was observed between the 2 sexes [IGF at $36 t_{(290)}=1.853 P=.24$ and IGF at $42 t_{(295)}=$ $1.148 P=.25]$. There appeared to be no significant correlations in males and females of IGF-I with physical activity and/or fitness in adolescence, and cognitive performance in adulthood (data not shown).

\section{Physical Activity in Adolescence and Cognitive Performance in Adulthood}

Multiple regression analyses were performed using physical activity in adolescence as a predictor for cognitive performance in adulthood.

Physical activity significantly predicted SWM Strategy and SWM BE in the group of all participants $(P<.001)$ Also, the interaction between sex and activity was significant $(P<.001)$. Separate regression analyses for males and females indicated 
Ferro, D.A., Deijen, J.B., Koppes, L.L., Mechelen, W. van, Twisk, J.W., Drent, M.L. The effects of physical activity and fitness in Adolescence on cognition in adulthood and the role of insulin like growth factor I . Journal of Physical Activity \& Health: 2016, 13(4), 392-402

that physical activity in adolescent males is a significant negative predictor for SWM BE and Strategy scores (Model 1) (Figure 1A and 1B). After correction for the covariates education, DART and physical activity in adulthood the association between physical activity in adolescence and SWM BE and Strategy scores are unchanged (Model 2). Finally, after also including IGF-I the association of physical activity in adolescence with SWM and Strategy Scores was still significant, while the association of physical activity in adolescence with SWM BE only tended to be significant (Model 3, $P=$.051) (Table 2).

No significant association was found between physical activity in adolescence and the SWM Strategy and SWM BE in females in any model (Model $1, P=.71$ and $P=$ .30 , respectively) (Table 3, Figure $1 \mathrm{~A}$ and $1 \mathrm{~B}$ ).

With respect to the PAL, in the total group of participants no significant association of physical activity in adolescence with the PAL TE and the PAL FTMS $(P=.74$ and $P=.69$, respectively) nor any moderator effect for sex was observed $(P=.43$ and $P$ $=.22$, respectively).

\section{[FIGURE 1][ TABLE 1]}

\section{Physical Fitness in Adolescence and Cognitive Performance in Adulthood}

A significant relationship between physical fitness and SWM Strategy and SWM BE was found in the group of all participants $(P<.001)$. Also a significant interaction between sex and physical fitness on SWM Strategy and SWM BE was found ( $P=$ .001 and $P=.03$, respectively). However, separate regression analyses in males and females did not result in a significant effect on SWM Strategy for males $(P=.22)$ or females $(P=.98)$ nor on SWM BE for males $(P=.09)$ or females $(P=.30)$ (Model 1) (Figure $2 \mathrm{~A}$ and $2 \mathrm{~B}$ ). Also after including education, DART and physical activity in adulthood as covariates (Model 2), and in addition IGF-I (Model 3), there was no significant association between physical fitness in adolescence and SWM BE and Strategy scores (Tables 2 and 3).

With respect to the PAL, in the total group of participants no significant association was found of physical fitness in adolescence with the PAL TE and the PAL FTMS $(P$ $=.41$ and $P=.91$, respectively), nor any moderator effect for sex was observed $(P=$ .91 and $P=.28$, respectively).

\section{DISCUSSION}

The present results indicate that physical activity in adolescence has different effects according to sex on cognitive performance in adulthood. Adult males who had been more physically active in adolescence, demonstrated better performance on the executive functions, working memory and strategic memory control than their peers who had previously been less active. No such relationship was found for females. Physical fitness in adolescence did not substantially predict cognitive performance in adult males, as only a trend for a relationship was found. No intermediate role for IGF-I was established in the relationship between physical activity or fitness and cognition.

Males who were more active in adolescence scored better on the SWM task in adulthood, a test assessing executive function which relies strongly on frontal lobe functioning. This finding is partly in agreement with a previous study reporting that men who had exercised (with low intensity) in adolescence, exhibited a higher information processing speed — strongly related to executive function ${ }_{43}$ - at old age 
Ferro, D.A., Deijen, J.B., Koppes, L.L., Mechelen, W. van, Twisk, J.W., Drent, M.L. The effects of physical activity and fitness in Adolescence on cognition in adulthood and the role of insulinlike growth factor I . Journal of Physical Activity \& Health: 2016, 13(4), 392-402

(62-85 years) than men who had not exercised.15 Although cognition was examined in elderly, as opposed to adults in our study, the studies are similar in finding longterm cognitive effects of adolescent physical activity.

The finding that executive functions benefit from physical activity during the adolescent years fits with the fact that the frontal lobes are still actively developing throughout adolescence, as is reflected by changes in brain tissue, particularly increases in white matter. This part of the brain is made of cells that are important for the transmission of signals to different parts of the brain. In several studies increased white matter density in the frontal lobe throughout adolescence has been demonstrated which is thought to underlie the development of executive function. 44,45 During adolescent years, activity might promote specific modifications to the frontal lobes, that could provide the basis of what we earlier described as cognitive reserve. As proposed by Stern, a cognitive reserve raises the threshold for clinical expression of brain-deterioration.6 Activity in adolescence could promote the building of a cognitive reserve, making these men more capable to cope with the age-related decline of executive function.

Neither physical activity, nor physical fitness in adolescence predicted performance in adulthood in either sex on the PAL, a visual-spatial memory task relying on medial temporal lobe functioning.

${ }_{33}$ One of the structures of the medial temporal lobe is the hippocampus, a brain area important for learning and memory.

Removal of new cells in the hippocampus has been found to result in spatial memory deficits,46 suggesting that the hippocampus plays an important role in spatial memory processes.

\section{[TABLE 2][TABLE 3][FIGURE 2]}

The present finding is not consistent with the evidence that in rodents exercise induces neurogenesis within the dentate gyrus of the hippocampus. 47 With respect to humans, an imaging study in elderly showed that having better physical fitness is also associated with increased hippocampal volume 48 and exercise training in the elderly halts age-related volume loss of the hippocampus.49 It may be that the PAL is not sensitive enough to detect subtle changes in hippocampal volume. Indeed, in a recent study, long-term aerobic exercise in healthy young adults was not found to improve the performance on the PAL. This result was explained by the evidence that the PAL is sensitive to hippocampal pathology, but not to hippocampal neurogenesis.50 No favorable effects of activity in adolescence on cognition were found for females. A previous study found an effect in women, 16 while other found that daily moderate-to-vigorous activity (MVPA) was associated with better executive function in adolescent males, with results less robust (ie, smaller regression coefficients) for females. In addition, the level of MVPA was found to be higher for males than for females. As a consequence, in boys, a 2 standard deviation increase in MVPA and in girls, a 3 standard deviation increase in MVPA was required to reach the recommended activity level of $60 \mathrm{~min}$ per day.14 Also in the current sample females appeared to be significantly less active than males. It could be that active males reached a certain threshold of activity - not obtained by the females - to promote the physiological changes underlying the improved frontal lobe function. Moreover, the physiological responses to physical activity may be different in males and females. 
Ferro, D.A., Deijen, J.B., Koppes, L.L., Mechelen, W. van, Twisk, J.W., Drent, M.L. The effects of physical activity and fitness in Adolescence on cognition in adulthood and the role of insulinlike growth factor I . Journal of Physical Activity \& Health: 2016, 13(4), 392-402

Recently, the increase in $\mathrm{VO}_{2}$ max after a 1-year endurance training was found to be markedly blunted in adult females compared with adult males.51 If this blunted response is also present in adolescents, it may be more difficult for adolescent females than for adolescent males to obtain the necessary physiological changes to improve brain function. However, as threshold of exercise intensity has been found to depend on initial fitness level, an exact threshold to improve cardiorespiratory fitness and/or other physiological or endocrinological parameters has not been established.52 The effect on cognition in adulthood was present more substantially for physical activity than physical fitness in adolescence. This finding is in agreement with former studies that found an association between cognition and low level physical activity — unlikely to reflect high aerobic fitness - and a stronger relationship of the amount of spare-time activity (eg, chess, bridge or similar games; playing a musical instrument with others) than actual physical exercise with memory.15,17 In addition, the cognition-enhancing effects of physical activity reported in a meta-analysis on 37 studies could not be explained by physical fitness. 7 As physical activity seems more important for cognition than physical fitness, it seems useful to mainly focus on physical activity while advocating a more healthy lifestyle. In addition, studies are needed to clarify the different mechanisms that play a role in the relationship between physical activity, fitness and cognition.

The current study assessed the role of IGF-I as an intermediate agent between physical activity and cognition. It was hypothesized that IGF-I release is stimulated by activity in adolescence and in turn could improve cognitive functioning in adulthood. No such long-term role for IGF-I was identified, for which several causes can be acknowledged. Firstly, a major drawback of this study is the fact that IGF-I levels were only measured in adulthood, whereas the building of a cognitive reserve was hypothesized to take place in adolescence. The assumption that epigenetic modifications of the IGF-I gene in adolescence may affect IGF-I release in adulthood may have been too speculative. Secondly, IGF-I blood levels may not reflect IGF-I levels in the brain. Actual cerebral IGF-I might be critical for the cognitive effects of exercise. For example, running in rats was found to enhance IGF-I levels in the brain, whereas serum IGF-I remained unaltered.22 However, as it is not possible to determine cerebral IGF-I activity in human brain, the determination of IGF-I in blood serum in humans is currently the most feasible method.

Our results on IGF-I are not in line with the finding that after 6 months of aerobic exercise in mildly cognitively impaired male and female subjects (55 to 85 years) IGF-I was increased in males.53 The lack of an effect of IGF-I is also opposed to the finding that a 12-month intervention of resistance exercise in healthy elderly males resulted in improved reaction times on a cognitive task that were associated with increased serum IGF-1 levels.54 However, in these 2 studies IGF-I was measured immediately following exercise.

One of the strengths of the current study lays in the prospective set up of objective measures of physical fitness throughout adolescence. In addition, in contrast with previous studies, applying robust cognitive tests such as the Mini-Mental State Examination (MMSE), 55 the current study was able to capture more subtle interpersonal differences using CANTAB subtests to assess visual-spatial memory and executive function. Finally, the cohort contained a reasonable amount of participants, allowing correction for confounders as previously identified in literature. 
Ferro, D.A., Deijen, J.B., Koppes, L.L., Mechelen, W. van, Twisk, J.W., Drent, M.L. The effects of physical activity and fitness in Adolescence on cognition in adulthood and the role of insulinlike growth factor I . Journal of Physical Activity \& Health: 2016, 13(4), 392-402

However, a number of limitations of the current study should be acknowledged. One of the limitations is the observational design which makes it difficult to infer causal relationships between variables.

Although an estimate of general cognitive ability (DART) was used as covariate, there were no appropriate baseline measures for executive functions and memory in adolescence. Therefore, it cannot be ascertained that early physical activity caused males to have better executive control in adulthood. Two alternative pathways should be considered. Firstly, reverse causality cannot be ruled out.

In other words, better executive control during adolescence caused these males to be more active, by for example successfully planning their day-to-day activities. Secondly, an underlying factor could be responsible for both activity and the development of executive control in adolescence. For instance, social economic status could potentially influence both factors as people from higher class backgrounds might be more aware of the benefits of physical activity and have had more opportunity to develop their executive functions. On the other hand, this study included a rather homogeneous sample of higher level secondary school pupils most likely of the same social background.

A further limitation is that the measures of physical activity were self-reported. However, the recollection of activities undertaken in the previous 3 months can be considered mildly retrospective.

Finally, the use of the PAL may be a limitation in the current study. Although the PAL is known to be hippocampal dependent, it may be inappropriate to detect changes in performance due to hippocampal neurogenesis. Maybe it would have been better to select a test that is specifically neurogenesis-dependent. One such a test is the visual pattern separation task developed by Kirwan and Stark.56 This task is based on the notion that the dentate gyrus of the hippocampus is responsible for pattern separation.56 As this task has a high interference component (ie, subjects have to discriminate between almost identical objects) and thus places high demands on pattern separation capabilities, it may be more useful than the PAL.

\section{CONCLUSIONS}

This prospective study reveals the longitudinal effects of physical activity in adolescence on cognition in adulthood. The present results indicate that adult males who have been more physically active in adolescence have a better working memory and strategic memory control than their peers who have previously been less active. Such a relationship between physical activity in adolescence and working memory and strategic memory control in adulthood was not found for females. In addition, physical fitness in adolescence tended to be associated with a better working memory in adulthood, but also only in males. These findings suggest that especially physical activity in adolescent men may improve frontal lobe-mediated cognitive functioning in adulthood.

In addition to the finding that physical fitness in adolescence is hardly involved in the relationship between physical activity and cognition, a role for IGF-I as an intermediate agent between physical activity and cognition could also not be confirmed.

Future studies are needed to further elucidate the mechanisms behind the relationship between physical activity in adolescence and better cognitive functioning in adulthood. It may be recommended to measure IGF-I at several time points during 
Ferro, D.A., Deijen, J.B., Koppes, L.L., Mechelen, W. van, Twisk, J.W., Drent, M.L. The effects of physical activity and fitness in Adolescence on cognition in adulthood and the role of insulinlike growth factor I . Journal of Physical Activity \& Health: 2016, 13(4), 392-402

adolescence to shed more light on its possible involvement in the building of a cognitive reserve. In addition, it seems useful to design studies that are directed on clarifying the different cognitive effects of physical activity in males and females. The findings of the current study contribute to the extensive area of research highlighting the importance of an active lifestyle in adolescence with respect to enduring healthy cognitive functioning.

\section{REFERENCES}

1. Caspersen CJ, Powell KE, Christenson GM. Physical activity, exercise, and physical fitness: definitions and distinctions for health-related research. Public Health Rep. 1985;100:126-131.

2. American Psychological Association. Glossary of psychological terms. ; 2013.

3. Blondell SJ, Hammersley-Mather R, Veerman JL. Does physical activity prevent cognitive decline and dementia?: A systematic review and meta-analysis of longitudinal studies. BMC Public Health. 2014;14:510 .

4. Hillman $\mathrm{Cl}$, Erikson $\mathrm{KI}$, Kramer AF. Be smart, exercise your heart: exercise effect on brain and cognition. Nat Rev Neurosci. 2008;9:58-65 .

5. Johansson MM, Marcusson J, Wressle E. Cognition, daily living, and health related quality of life in 85-year-olds in Sweden. Neuropsychol Dev Cogn B Aging Neuropsychol Cogn. 2012;19:421-432.

6 . Stern $Y$. What is cognitive reserve. Theory and research applications of this reserve concept. J Int Neuropsychol Soc. 2002;8:448-460.

7. Etnier JL, Nowell PM, Landers DM, Sibley BA. A meta-regression to examine the relationship between aerobic fitness and cognitive performance. Brain Res Brain Res Rev. 2006;52:119-130.

8. Richards M, Deary IJ. Cognitive reserve: a model for cognitive aging and development? Ann Neurol. 2005;58:617-622 . 9. Stern Y. Cognitive reserve in ageing and Alzheimer's disease. Lancet Neurol. 2012;11:1006-1012 .

10. Colcombe SJ, Erickson KI, Raz N, et al. Aerobic fitness reduces brain tissue loss in aging humans. J Gerontol A Biol Sci Med Sci. 2003;58A:176-180.

11. Lindwall M, Cimino CR, Gibbons LE, et al. Dynamic associations of change in physical activity and change in cognitive function: coordinated analyses of four longitudinal studies. J Aging Res. 2012;2012:1- 12.

12. Robinson G, Shallice T, Bozzali M, Cipolotti L. The differing roles of the frontal cortex in fluency tests. Brain. 2012;135:2202-2214 .

13. Donnally JE, Lambourne K. Classroom-based physical activity, cognition, and academic achievement. Prev Med. 2011;52:S36-S42 . 14. Booth JN, Tomporowski PD, Boyle JM, et al. Associations between executive attention and objectively measured physical activity in adolescence: Findings from ALSPAC, a UK cohort. Ment Health Phys Act. 2013;6:212219.

15. Dik MG, Deeg DJH, Visser M, Jonker C. Early life physical activity and cognition at old age. J Clin Exp Neuropsychol. 2003;25:643-653 .

16. Middleton LE, Barnes DE, Lui LY, Yaffe K. Physical activity over the life course and its association with cognitive performance and impairment in old age. J Am Geriatr Soc. 2010;58:1322.

17. Richards M, Hardy R, Wadsworth MEJ. Does active leisure protect cognition. Evidence from a national birth cohort. Soc Sci Med. 2003;56:785-792 .

18. Singh-Manoux A, Hillsdon M, Brunner E, Marmot M. Effects of physical activity on cognitive functioning in middle age: evidence from the Whitehall II prospective cohort study. Am J Public Health. 2005;95:2252-2258 .

19. Miyake A, Friedman NP, Emerson MJ, Witzki AH, Howerter A, Wager TD. The unity and diversity of executive functions and their contributions to complex "frontal lobe" tasks: a latent variable analysis. Cognit Psychol. 2000;41:49-100 . 
Ferro, D.A., Deijen, J.B., Koppes, L.L., Mechelen, W. van, Twisk, J.W., Drent, M.L. The effects of physical activity and fitness in Adolescence on cognition in adulthood and the role of insulinlike growth factor I . Journal of Physical Activity \& Health: 2016, 13(4), 392-402

20. Chang M, Jonsson PV, Snaedal J, et al. The effect of midlife physical activity on cognitive function among older adults: AGES-Reykjavik Study. J Gerontol A Biol Sci Med Sci. 2010;65A:1369-1374 .

21. Sofi F, Valecchi D, Bacci D, et al. Physical activity and risk of cognitive decline: a metaanalysis of prospective studies. J Intern Med. 2011;269:107-117.

22. Carro E, Nunez A, Busiguina S, Torres-Aleman I. Circulating insulinlike growth factor I mediates effects of exercise on the brain. J Neurosci. 2000;20:2926-2933.

23. Deijen JB, Van Driel MI, Drent ML. The involvement of the GH/IGF-I Axis in cognitive functions of adult patients and healthy subjects. Open

Endocrinol J. 2012;6:68-79 .

24. Frystyk J. Exercise and growth hormone-insuline-like growth factor axis. Med Sci Sports Exerc. 2009;42:58-66 .

25. Eliakim A, Brasel JA, Mohan S, Barstow TJ, Berman N, Cooper DM. Physical fitness, endurance training, and the growth hormone-insulinlike growth factor I system in adolescent females. J Clin Endocrinol Metab. 1996;81:3986 10.1210/jcem.81.11.8923848.

26. Cosín-Tomás M, Alvarez-López MJ, Sanchez-Roige S, et al. Epigenetic alterations in hippocampus of SAMP8 senescent mice and modulation by voluntary physical exercise. Front Aging Neurosci. 2014;6:51 10.3389/fnagi.2014.00051.

27. Fu Q, Yu X, Callaway CW, Lane RH, McKnight RA. Epigenetics: intrauterine growth retardation (IUGR) modifies the histone code along the rat hepatic IGF-1 gene. FASEB J. 2009;23:2438-2449 .

28. Ntanasis-Stathopoulos J, Tzanninis J-G, Philippou A, Koutsilieris M. Epigenetic regulation on gene expression induced by physical exercise. J Musculoskelet Neuronal Interact. 2013;13:133-146.

29. Kemper HCG, Borms J, Hebbelinck M, Hills AP. Amsterdam Growth and Health Longitudinal Study (AGAHLS); A 23-Year Follow-up From Teenager to Adult About Lifestyle and Health. Medicine and Sport Science, 47. Basel: Karger; 2004.

30. Kemper HCG, Van Mechelen W. Methods of measurement used in the longitudinal study. In: Kemper HCG, ed. The Amsterdam Growth and Health study: A Longitudinal Analysis of Health, Fitness and Lifestyle. HK Sports Science Monograph Series. Campaign, IL: Human Kinetics; 1995:28-49.

31. Van Mechelen W, Twisk JWR, Post GB, Snel J, Kemper HCG. Physical activity of young people: the Amsterdam Longitudinal Growth and Health Study. Med Sci Sports Exerc. 2000;32:1610-1616.

32. Montoye HJ, Kemper HCG, Saris WHM, Washburn RA. Measuring Physical Activity and Energy Expenditure. Campaign, IL: Human Kinetics; 1996.

33. Robbins TW, James M, Owen AM, Sahakian BJ, McInnes L, Rabbitt PM. Cambridge Neuropsychological Test Automated Battery (CANTAB): a factor analytic study of a large sample of normal elderly volunteers. Dementia. 1994;5:266-281.

34. CANTABeclipse. Test Administration Guide. Cambridge, UK: Cambridge Cognition Limited; 2011.

35. Nelson HE, Wilson JR. The Revised National Adult Reading Test-Test Manual. Winsor: NFER-Wilson; 1991.

36. Schmand B, Smit JH, Geerlings MI, Lindeboom J. The effects of intelligence and education on the development of dementia. A test of the brain reserve hypothesis. Psychol Med. 1997;27:1337-1344.

37. Van Mechelen W, Kemper HCG. Habitual physical activity in longitudinal perspective. In: Kemper HCG, ed. The Amsterdam Growth and Health study: A Longitudinal Analysis of Health, Fitness and Lifestyle. HK Sports Science Monograph Series. Campaign, III: Human Kinetics; 1995:135-158.

38. Fox-Wasylyshyn SM, El-Masri MM. Handling missing data in self-report measures. Res Nurs Health. 2005;28:488-495. 39. Grau E, Ahmed S. Evaluation of imputation of covariates in an impact analysis with regression adjustment. JSM Proceedings, Health Policy

Statistics Section. American Statistical Association; 2008;114-124.

40. Maggio M, Ble A, Ceda GP, Metter EJ. Decline in Insulin-like growth factor-I levels across adult life span in two large population studies. J Gerontol A Biol Sci Med Sci. 2006;61:182-183. 
Ferro, D.A., Deijen, J.B., Koppes, L.L., Mechelen, W. van, Twisk, J.W., Drent, M.L. The effects off physical activity and fitness in Adolescence on cognition in adulthood and the role of insulin-

like growth factor I . Journal of Physical Activity \& Health: 2016, 13(4), 392-402

41. Cook C. Clinimetrics corner: use of effect sizes in describing data. J Manual Manip Ther. 2008;16:54E-57E .

42. Baron RM, Kenny DA. The moderator-mediator variable distinction in social psychology research: conceptual, strategic and statistical considerations. J Pers Soc Psychol. 1986;51:1173-1182.

43. Salthouse TA. Relations between cognitive abilities and measures of executive functioning. Neuropsychology. 2005;19:532-545. 44. Giedd JN, Rapoport JL. Structural MRI of pediatric brain development: what have we learned and where are we going? Neuron. 2010;67:728-734 .

45. Blakemore S, Choudhury S. Development of the adolescent brain: implications for executive function and social cognition. J Child Psychol Psychiatry. 2006;47:296-312 .

46. Imayoshi I, Sakamoto M, Ohtsuka T, et al. Roles of continuous neurogenesis in the structural and functional integrity of the adult forebrain. Nat Neurosci. 2008;11:1153-1161

47. Van Praag $\mathrm{H}$. Exercise and the brain: something to chew on. Trends Neurosci. 2009;32:283-290.

48. Erickson KI, Prakash RS, Voss MW, et al. Aerobic fitness is associated with hippocampal volume in elderly humans. Hippocampus. 2009;19:1030-1039 .

49. Erickson KI, Voss MW, Prakash RS, et al. Exercise training increases size of hippocampus and improves memory. Proc Natl Acad Sci USA. 2011;108:3017-3022 .

50. Déry N, Pilgrim M, Gibala M, et al. Adult hippocampal neurogenesis reduces memory interference in humans: opposing effects of aerobic exercise and depression. Front Neurosci. 2013;7:66 .

51. Howden EJ, Perhonen M, Peshock RM, et al. Females have a blunted cardiovascular response to 1-year of intensive supervised endurance training. J Appl Physiol (1985). 2015;119(1):37-46.

52. Garber CE, Blissmer B, Deschenes MR, et al. American College of Sports Medicine position stand. Quantity and quality of exercise for developing and maintaining cardiorespiratory, musculoskeletal, and neuromotor fitness in apparently healthy adults: guidance for prescribing exercise. Med Sci Sports Exerc. 2011;43:1334-1359 .

53. Baker LD, Frank LL, Foster-Schubert K, et al. Effects of aerobic exercise on mild cognitive impairment: a controlled trial. Arch Neurol. 2010;67:71-79 .

54. Tsai $\mathrm{CL}$, Wang $\mathrm{CH}$, Pan $\mathrm{CY}$, Chen FC. The effects of long-term resistance exercise on the relationship between neurocognitive performance and GH, IGF-1, and homocysteine levels in the elderly. Front Behav Neurosci. 2015;9:23 .

55. Folstein MF, Folstein SE, McHugh PR. "Mini-mental state." A practical method for grading the cognitive state of patients for the clinician. J Psychiatr Res. 1975;12:189-198 .

56. Kirwan $\mathrm{CB}$, Stark $\mathrm{CE}$. Overcoming interference: an $\mathrm{fMRI}$ investigation of pattern separation in the medial temporal lobe. Learn Mem. 2007;14:625-633 . 
Ferro, D.A., Deijen, J.B., Koppes, L.L., Mechelen, W. van, Twisk, J.W., Drent, M.L. The effects of physical activity and fitness in Adolescence on cognition in adulthood and the role of insulin-

\section{TABLES AND FIGURES}

\section{Table 1 Mean (SD) Physical Activity, Fitness, and Cognitive Scores}

\begin{tabular}{lcc}
\hline & Males $(\mathbf{n}=143)$ & Females $(\mathbf{n}=160)$ \\
\hline Physical activity (MET/week) & $4454(1622)$ & $3637(1638)$ \\
Adolescence* & $2669(735)$ & $2838(966)$ \\
Adulthood & & \\
Aerobic fitness $\left(\mathrm{VO}_{2}\right.$ max, $\left.\mathrm{ml} / \mathrm{min}^{-1} / \mathrm{kg}^{-2 / 3}\right)$ & $59.32(4.85)$ & $49.31(5.05)$ \\
Adolescence* & $51.58(6.88)$ & $40.86(6.17)$ \\
Adulthood* & & \\
Cognition (CANTAB) & $12.73(12.35)$ & $20.15(14.31)$ \\
SWM between errors* & $27.64(6.55)$ & $31.27(5.91)$ \\
SWM strategy* & $14.38(3.00)$ & $14.40(2.75)$ \\
PAL first trial memory score & $1.06(.34)$ & $1.01(.34)$ \\
PAL total errors (logarithm) & $15.23(13.09)$ & $13.07(10.00)$ \\
PAL total errors & & \\
IGF-I (nmol/l) & $26.51(5.47)$ & $25.10(6.17)$ \\
Mean age 36 & $24.27(5.45)$ & $23.39(5.46)$ \\
Mean age 42 & & \\
\hline
\end{tabular}

Abbreviations: DART, Dutch Adult Reading Test; SWM, spatial working memory; PAL, Paired associate learning. $* P<.0001 ;$ males vs females. 
Ferro, D.A., Deijen, J.B., Koppes, L.L., Mechelen, W. van, Twisk, J.W., Drent, M.L. The effects off physical activity and fitness in Adolescence on cognition in adulthood and the role of insulin-

like growth factor I . Journal of Physical Activity \& Health: 2016, 13(4), 392-402
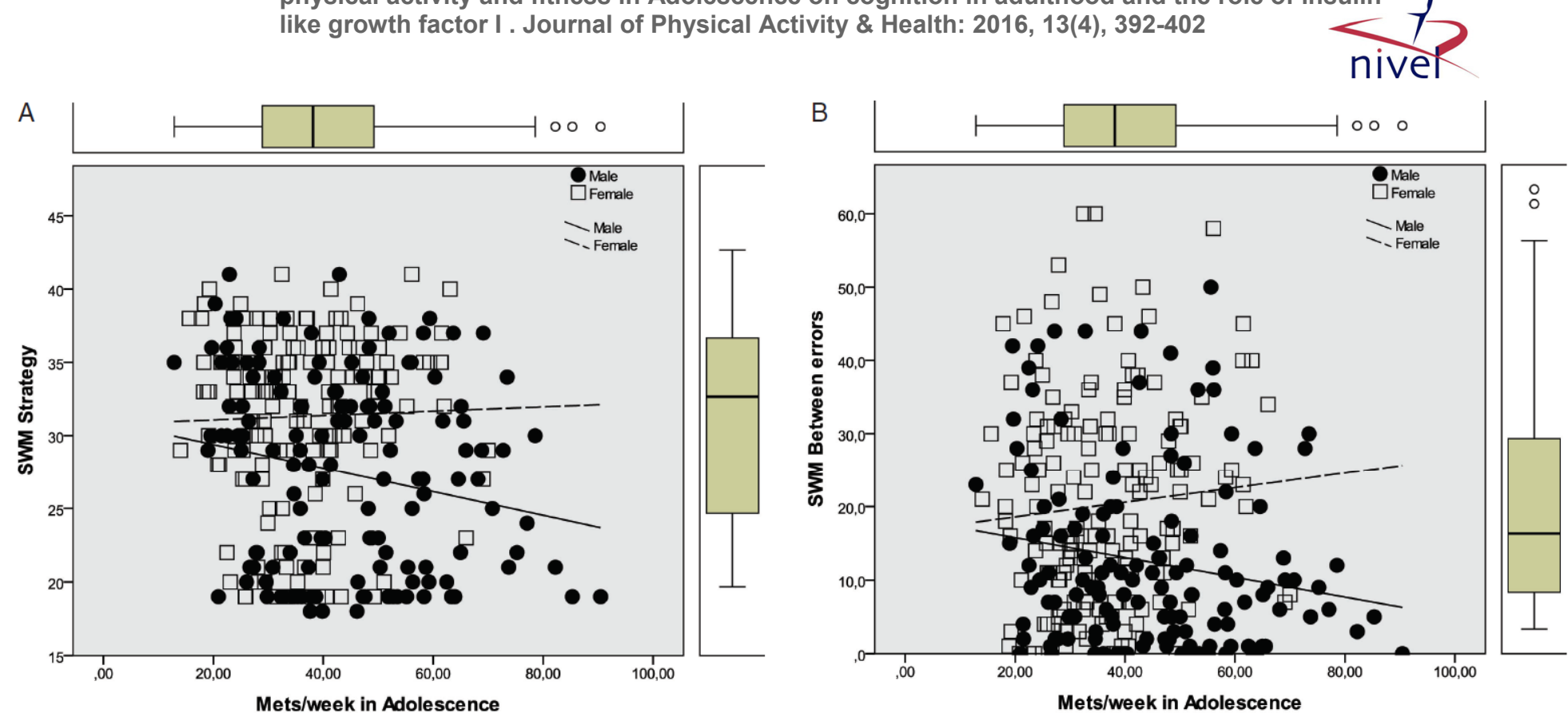

Figure 1 - A and B: Linear regression of physical activity in adolescence on SWM (Spatial Working Memory) Strategy and SWM Between Errors in adulthood in males and females. Lower scores indicate better performance (males: $P=.02$ and $P=.03$, respectively). Solid line represents regression line for males, dashed line for females. 
Ferro, D.A., Deijen, J.B., Koppes, L.L., Mechelen, W. van, Twisk, J.W., Drent, M.L. The effects of physical activity and fitness in Adolescence on cognition in adulthood and the role of insulin-

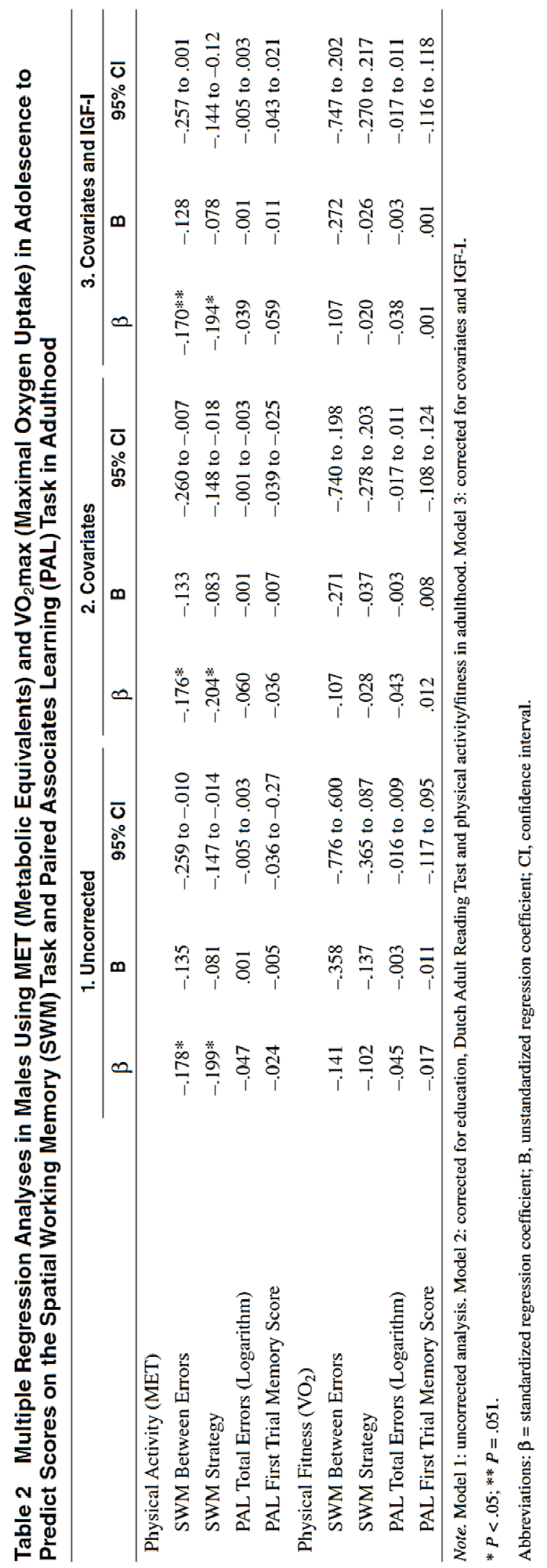


Ferro, D.A., Deijen, J.B., Koppes, L.L., Mechelen, W. van, Twisk, J.W., Drent, M.L. The effects of physical activity and fitness in Adolescence on cognition in adulthood and the role of insulin-

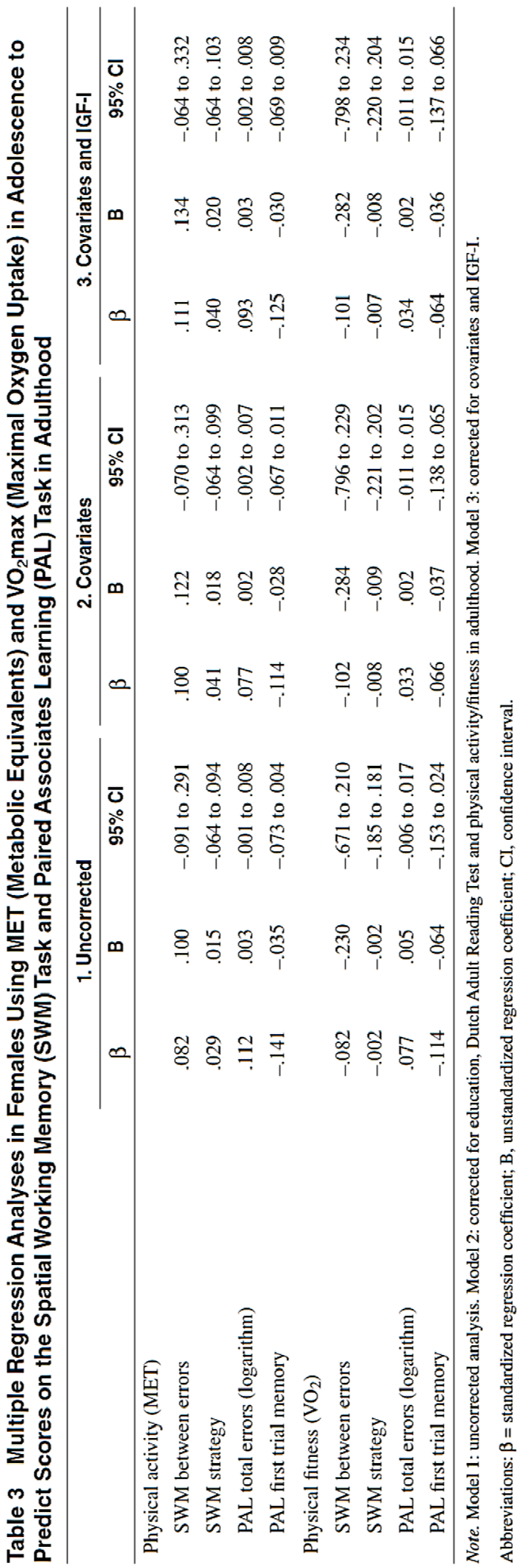


Ferro, D.A., Deijen, J.B., Koppes, L.L., Mechelen, W. van, Twisk, J.W., Drent, M.L. The effects of physical activity and fitness in Adolescence on cognition in adulthood and the role of insulin-

like growth factor I . Journal of Physical Activity \& Health: 2016, 13(4), 392-402
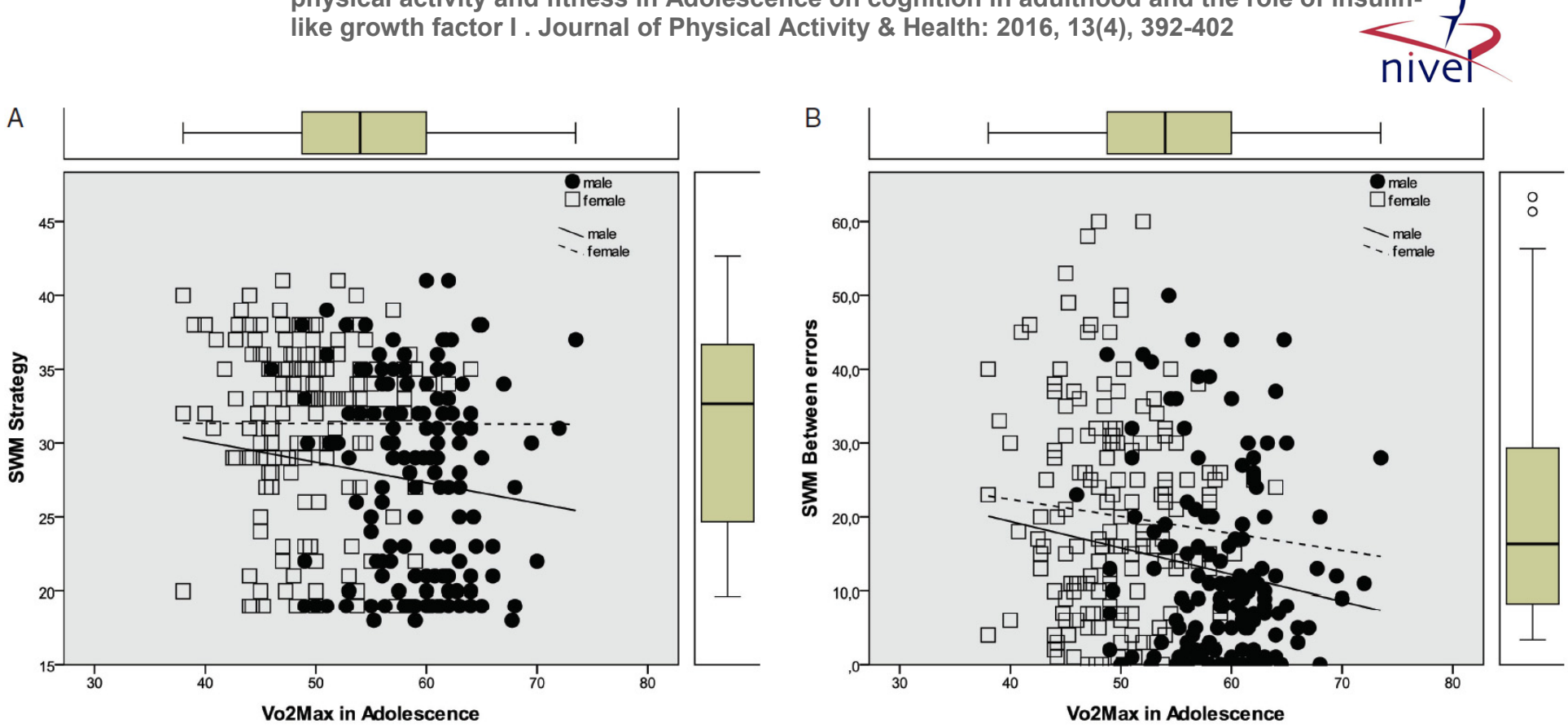

Figure 2 - A and B: Linear regression of physical fitness in adolescence on SWM (Spatial Working Memory) Strategy and SWM Between Errors in adulthood in males and females. Lower scores indicate better performance (males: $P=.22$ and $P=.09$, respectively). Solid line represents regression line for males, dashed line for females. 